Synthetic Polymeric Membranes, Editors: B. Sedláček, J. Kahovec // Walter de Gruyter \& Co. Berlin

-New York - Printed in Germany 1987 c.363-375

\title{
USE OF COMPUTING PROGRAMMES FOR EVALUATING RESULTS OF DIFFUSION EXPERIMENTS
}

\author{
I.N. Beckman, A.A. Shviryaev and V. Balek
}

\section{Introduction}

New synthetic polymers, e.g. block copolymers and polymer composite materials, containing adsorbents, catalysts, etc. have been applied recently in the technology of synthetic polymer membranes.

Anomalies of permeability and diffusion of penetrating agents in synthetic membranes encountered in experiments may have their origin in interactions between components of the penetrating agents as well as in an interaction of the penetrating agents with various structure formations in hard polymeric materials. In these cases the permeability, solubility and diffusion properties of polymer membranes may strongly depend on the concentration of the penetrating agent, time and the local structure properties of the polymeric materials.

Obviously, for the preparation of polymer membranes of required properties we have to know the mechanism of migration of the penetrating agent in the materials of membranes. This information can be obtained by several methods checking the transport properties, e.g. permeability, adsorption, desorption of gases, thermodesorption spectra and other methods. In this way a relatively large number of experimental data are obtained, which must be evaluated, compared with theoretical diffusion models and eventually stored for further use.

Use of rapid computers enabled us to collect and store all experimental data of the methods applied, to construct a theoretical model of diffusion, and to evaluate the diffusion parameters of the polymeric material. We have prepared a set of computer programmes called DIGS (diffusion of inert gases in solids) for the statistical treatment and interpretation of the results of various methods used for the investigation of transport properties of polymeric materials. The theoretical models of approx. 60 diffusion cases are stored in the memory of the computer, as input parameters being considered (1):

- the concentration distribution along the width of the sample

- the quantity of the penetrating substance in the solid, $G(t)$

- the quantity of the penetrating substance in the gaseous phase, $M(t)$

- the quantity of the penetrating substance which has passed through the membrane, $q(t)$

- the diffusion fluxes at the input and output of the sample, $Q(t)$

Various geometrical shapes (sphere, cylinder, plate) can be taken into account; diffusion coefficients depending on time, concentration and coordinates of the sample can be evaluated. Other diffusion parameters can be determined and the fitness of adequate diffusion models can be verified $(1,2)$.

The set of computer programmes DIGS is prepared in the Fortran language and can be easily adapted to any computer equipped with a Fortran translator.

A bank of general diffusion models has been organized on the basis of both analytical and numerical solutions of diffusion cases. In the bank of experimental data the standard interface for the input of experimental data is used.

The set of the programmes involves four parts which give the following possibilities:

(1) preliminary treatment of experimental results, 
(2) evaluation of the results on the basis of the classical diffusion model,

(3) choice of a more suitable phenomenological diffusion model (in case that the classical diffusion model is inadequate),

(4) choice of a suitable physical diffusion model.

\section{Preliminary Treatment of Experimental Results}

One of the main tasks of this part of the programme is to eliminate apparatus errors, as the choice of suitable diffusion models requires knowledge of the true form of the kinetic curves of diffusion. Unfortunately, in the course of the registration of the diffusion flux in the experimental measuring system a distortion of the input signal takes place caused by the integration effect of the detector employed, as well as by the inertia of the apparatus, due to the adsorption of the penetrating agent on the walls of the apparatus, etc.

Consequently, in real experiments, especially when using so-called rapid membranes, characterized by a high diffusion coefficient and low thickness of the membranes, a distorted curve results.

The diffusion apparatus is tested in order to estimate the distortion effect of the apparatus on the experimental curve. The testing consists in measuring the apparatus response to a short impulse of the penetrating agent.

The resulting apparatus response $\phi(t)$ is usually normalized with respect to the total amount of the penetrating agent; the true form of the experimental curve can be obtained by solving the Fredholm equation of the first order

$$
f(t)=\int_{0}^{t} \phi(t-\tau) y(\tau) \mathrm{d} \tau
$$

where $f(t)$ is the experimentally recorded curve, $y(t)$ is the true curve, $\phi(\mathrm{t})$ is the response of the apparatus to the short-time pulse.

In Fig. 1, curves 1, 2, and 3 correspond to curves $\phi(t), f(t)$ and $y(t)$, resp., in the gas permeability experiment. The existence of statistical errors in the experiment impedes the finding of an exact solution to Eq.(1) and renders the task incorrect. The solution to equation (1) has been found by the one-parameter regularization methods (2) only within the accuracy of experimental measurements.

As follows from Fig.1, curves 2 and 3, the inertia, of the apparatus causes an important distortion of the experimental curve, a time delay and a decrease in the flux $Q(t)$ of the penetrating agent through the membrane. Unfortunately, when systematic errors of the experiments are taken into account, the statistical errors of the experiments increase. Consequently, it is necessary to use a dynamic and precise apparatus for the diffusion measurements in membrane materials.

\section{Evaluation of Experimental Results on the Basis of the Classical Diffusion Model}

After the reconstruction of true experimental curves the diffusion parameters can be evaluated. Several methods are discussed here.

The method of linearization $(3,4)$ is based on the application of functional scales. For the results of gas permeability measurements the following expression can be used to construct the functional scale:

$$
y(t)=y_{0} \mathrm{~F}\left\{D l^{-2}\left(t-t_{\mathrm{o}}\right)\right\}=y_{0} F(U)
$$

where $t$ is time of the onset of diffusion, $U=D t / l^{2}$, and the function $F(U)$ is given by Eq.(3) 


$$
F(U)=2(\pi U)^{-1 / 2} \sum_{n=1}^{\infty} \exp \left[-(n-0.5)^{2} / U\right]=1+2 \sum_{n=1}^{\infty}(1)^{n} \exp \left(-n^{2} \pi^{2} U\right)
$$

By means of Eq.(3) the function $F(U)$ can be evaluated; equal intervals of the function $F(U)$ are established and the corresponding values of $U$ are found. Both values of $U$ and $F(U)$ are plotted on the axis of ordinates, the former on the right, the latter on the left. Time $t$ is plotted on the axis of abscissae.

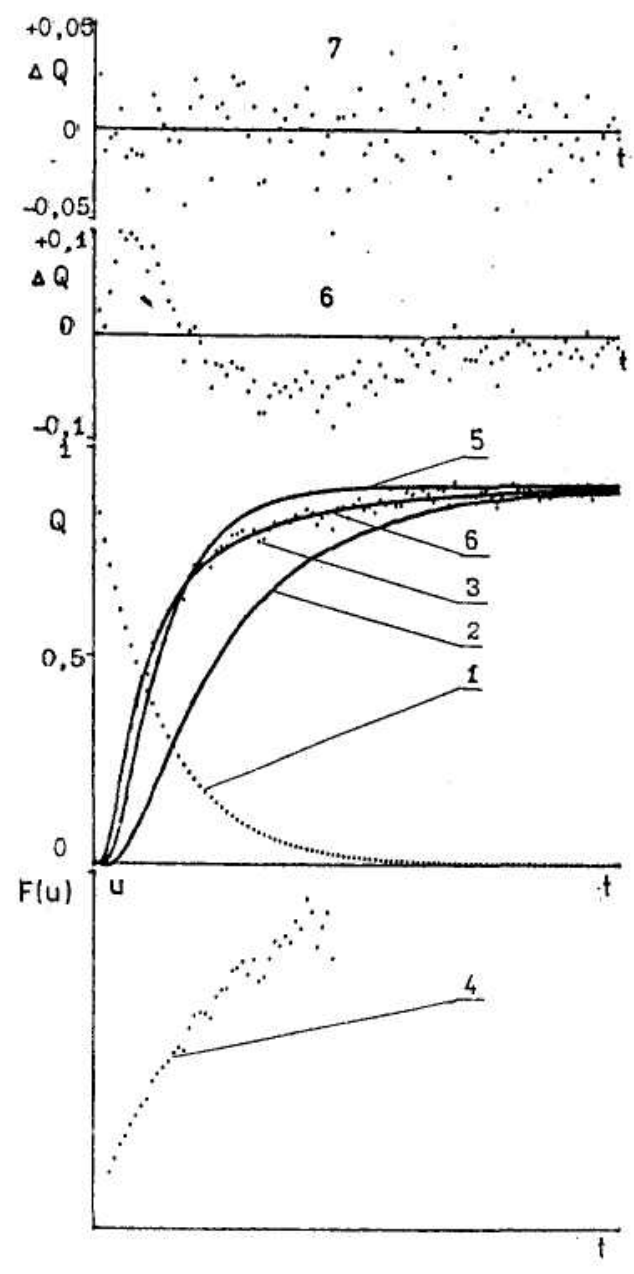

Fig.1. Evaluation of the measurements of gas permeability of polymer membranes. The curves are marked as follows: 1 - response of the apparatus to a gas impulse; 2 - experimental curve representing the time dependence of the inertia of the apparatus; 3 - reconstructed experimental points; 4 - curve 3 plotted on a functional scale; 5 - computed curve based on the classical diffusion mechanism (non-adequate for this case); 6 - computed curve based on the parallel diffusion mechanism (adequate for this case) ; 7 -drift of residues from the base line corresponding to curve $5 ; 8$ - drift of residues from the base line corresponding to curve 6 .

Using the functional scale constructed in this way the experimental values are normalized with respect to the steady-state gas flux; i.e. $F(U)=Q(t) / \mathrm{Q}_{\infty}$. Curve 4 in Fig. 1 represents the example of a linearized curve obtained from gas permeation experiments, curve 3 in Fig. 1 represents the true experimental curve on a normal scale. The linear dependence in Fig.1, curve 4, is described by the function $U=\left(D / l^{2}\right)$. $\left(t-t_{0}\right)$, the diffusion coefficient $D$ can be evaluated from $D=l^{2} x \operatorname{tg} \alpha$, where $\operatorname{tg} \alpha$ is the slope of the linearized dependence. 
From curve 4 in Fig. 1 we have that gas diffusion in the membrane is characterized by two different values of the diffusion coefficient which correspond to the model of the diffusion through two independent channels.

Typical experimental curves of various gas permeability methods are demonstrated in Figs.2a-d, namely (a) the integral method, (b) the differential method, (c) the impulse method and (d) the differential impulse method.

Using experimental data of these methods, a preliminary estimation of diffusion parameters can be performed by the method of particular points.

With the integral method the point $\theta$ (see Fig.2a), determined by an extrapolation of the linear part of the curve to its cross-section point with the axis of abscissae, can be used. With the differential method the point $\tau, 1 / 2$ (see Fig.2b), corresponding to the half concentration of the gas at the membrane input, can be used. When the impulse and differential pulse methods are used, the points $\tau_{\mathrm{m}}$ and $\tau_{\mathrm{i}}$ can be determined from the respective experimental curves (see Fig.2c,d). Any one among the particular points can be used for the determination of the diffusion coefficient $D$, using Eq.(4)

$$
D=\frac{l^{2}}{19.9 \tau_{i}}=\frac{l^{2}}{10.9 \tau_{m}}=\frac{l^{2}}{7.2 \tau_{1 / 2}}=\frac{l^{2}}{6 \theta}
$$

supposing that

$$
Q=\frac{Q\left(\tau_{m}\right)}{0.2442}=\frac{Q\left(\tau_{1 / 2}\right)}{0.5}=\frac{Q(\theta)}{0.6266}
$$

where $l$ is the thickness of the membrane.

The homogeneity of the diffusion medium can simply be estimated by means of the "particular points". For a homogeneous polymer membrane all values of the diffusion coefficient calculated from Eq. (4) are identical.

For non-homogeneous membranes, where some diffusion paths lead to enhanced diffusion and to short diffusion times, the values of D calculated from Eq.(4) differ.

A rapid determination of the diffusion coefficient can be made (even before the steady state is attained) by means of the particular point $\tau$, as follows from Eq.(6)

$$
D=\frac{l^{2} \operatorname{tg} \alpha}{24.414 Q\left(\tau_{m}\right)} ; \quad Q_{\infty} \frac{Q\left(\tau_{m}\right)}{0.2442}
$$

where tg $\alpha$ is the slope of the linear part of the curve extrapolated to the point $\tau_{\mathrm{m}}$.

Method of statistical parameters. This method is based on the supposition that any function depending on time (and tending to a constant value at $t \rightarrow \infty$ ) can be considered as the integral distribution function of an accidental parameter. This statistical approach enabled us to understand the physical meaning of the "particular points".

Any limited normalized function $y=f(t)\left(\mathrm{y} \geq 0, \lim _{t \rightarrow \infty} y=0\right)$ may be considered as the density of an accidental parameter; in the case of the pulse method of the gas permeability measurement the density of the distribution can be described by Eq. (7)

$$
f=\mathrm{d} F / \mathrm{d} t=2 \sum_{n=1}^{\infty}(-1)^{n} n^{2} \pi^{2} D l^{-2} \exp \left\{-\left(n \pi / l^{2}\right) D t\right\}
$$




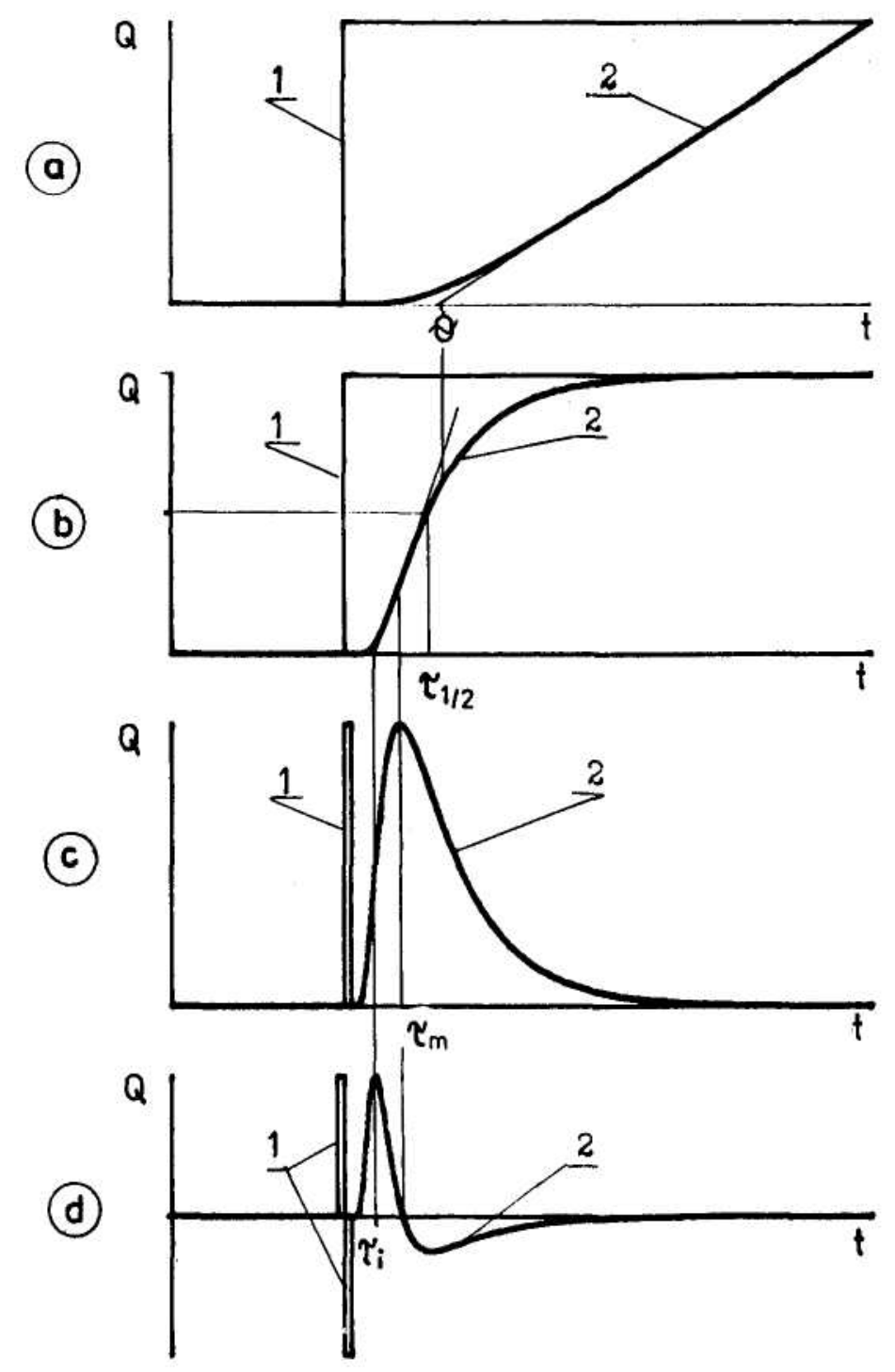

Fig.2. Typical experimental curves resulting from various gas permeation methods (curves 1 and 2 correspond to the time dependence of partial pressure of the diffusion agent on the membrane input and that of the gas flux through the membrane, respectively): a) integral method, b) differential method, c) impulse method, d) differential impulse method.

The integral function $F(t)$, representing the normalized gas flux, is used for the determination of the statistical parameters $\mu_{\mathrm{k}}$ according to Eq.(8)

$$
\mu_{k}=\int_{0}^{\infty} F(\tau) \mathrm{d} \tau^{k}=\int_{0}^{\infty} f(\tau) \tau^{k} \mathrm{~d} \tau
$$

The universal way for determining the statistical parameters consists in an integration of the experimental curves, plotted as the dependence of $Q$ against $t, t^{2}, t^{3}$, etc.

It should be pointed out that the experimental results must be presented as a set of numerical data (instead of a graphic plot). 
The following expressions are valid for the statistical feed parameters of the pulse gas permeability curves: $\mu_{1}=l^{2} / 6 \mathrm{D}=\theta$ - this parameter is called the mean value of the curve; $\mu_{2}=(7 / 5) \theta^{2}, \mu_{3}=(93 / 35) \theta^{3}$, $\mu_{4}=(1143 / 175) \theta^{4}$.

The central statistical parameters of the curves are defined as

follows: $M_{2}=(2 / 5) \theta^{2} \quad$ curve dispersity

$$
\begin{aligned}
& M_{3}=(16 / 35) \theta^{3} \quad \text { curve asymmetry } \\
& M_{4}=(228 / 175) \theta^{4} \text { curve excess }
\end{aligned}
$$

By means of the statistical parameters a full description of the pulse gas permeability curve can be made. The more generalized description of the curve is possible by means of the basic statistical parameters $\beta_{1}$ and $\beta_{2}$, representing the central statistical parameters of asymmetry $M_{3}$ and excess $M_{4}$ normalized with respect to the parameters $M_{2}$ - dispersity, i.e. $\beta_{1}=M_{3} / M_{2}=160 / 49=3.27 ; \beta_{2}=M_{4} / M_{2}=57 / 7=8.14$.

In this way, using the coordinates $\beta_{1}$ and $\beta_{2}$, all curves of the gas permeability measurements can be transformed into one point only, determined by the co-ordinates $\beta_{1}=3.27$ and $\beta_{2}=8.14$. In Fig. 3 the diagram called the "diffusion map" is demonstrated, the point discussed above being designated by number $\underline{6}$.

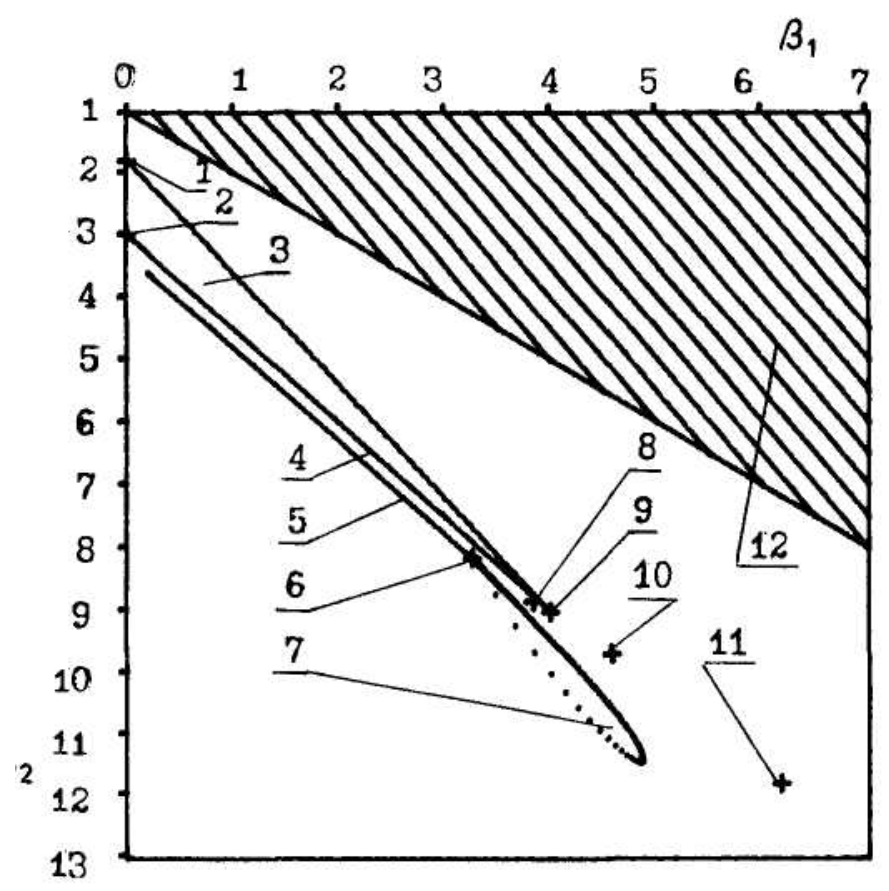

Fig.3. Diffusion map representing various cases of gas permeability of polymer membranes: 1 - uniform distribution; 2 - normal distribution; 3 - region of beta distribution; 4 - gamma-distribution; 5 - permeability model plus an irreversible chemical reaction of the 1st order between the gas and traps of the membrane (permanent trapping); 6 permeability model; 7 - permeability model based on the parallel diffusion; 8 - permeability measured by the electrochemical method; 9 - exponential distribution (single jump diffusion, kinetic stage of diffusion).

It is obvious that the expressions for the statistical parameters are much simpler than the initial expressions describing the diffusion kinetics. Hence, the application of the method of statistical parameters in an evaluation of diffusion and related parameters is rapid and easy.

Examples of evaluations are given below. In the polymer membrane where reversible interactions of the penetrating agent with defects of the material take place, the time dependence of the gas permeability can be described by Eq. (9) 


$$
Q(t)=C_{0} D S l^{-1}\left\{1-\sum_{n=1}^{\infty}\left[(-1)^{n} / A\right]\left[\left(\alpha_{1}-k_{1}-k_{2}\right) \exp \left(\alpha_{1} t\right)-\left(\alpha_{2}-k_{1}-k_{2}\right) \exp \left(\alpha_{2} t\right)\right]\right\}
$$

where $\alpha_{1}=0.5\left(k_{1}+k_{2}+D w\right)-A, \alpha_{2}=0.5\left(k_{1}+k_{2}+D w\right)+A, A=k_{1} k_{2}+0.25\left(k_{1}+k_{2}+D w\right) ; w=n \pi / l ; C_{0}$ is the input concentration, $S$ - surface area of the membrane, $k_{1}$ probability of gas trapping on a defect, $k_{2}$ probability of gas escape from defect; $K=k_{1} / k_{2}$.

The expressions for the statistical parameters are, however, simpler: $M_{1}=\theta=(1+K) /(6 B)$; $\left.M_{2}=7(1+K)^{2} /\left(180 B^{2}\right)+K /\left(3 k_{2} B\right) ; M_{3}=31(1+K)^{3} /\left(2520 B^{3}\right)+7 K(1+K) /\left(120 k_{2} B^{2}\right)+K / k_{2}^{2} B\right)$.

The determination of the first three statistical parameters of the experimental curve enabled us to evaluate all parameters of gas diffusion by a simultaneous 1st-order chemical reaction between the gas and the traps of the solid, i.e. $B=D / l^{2}, k_{1}, k_{2}$ and $K$.

The statistical parameters are evaluated by computers, based on the supposition of the classical diffusion mechanism. The nonlinear way of application of the least square method is suitable for an evaluation of diffusion data and their errors. An example of this application is given in Fig.1.

\section{Choice of a More Suitable Phenomenological Model of Gas Diffusion}

In the case that the experimental data treated so far do not correspond to the model of classical diffusion, another more adequate diffusion model has to be chosen for the determination of the diffusion coefficient. The basic expressions necessary for the computer treatment are stored in a special bank of phenomenological diffusion models. The choice of an adequate diffusion model is made by a step-wise trial of various models, starting from a simple to more complicated ones.

The general equation giving the possibility of describing various diffusion situations and diffusion models is given in Eq.(10)

$$
\frac{\partial C}{\partial t}=\frac{\partial}{\partial x} D(c, x, t) \frac{\partial C}{\partial x}+f(c, x, t)
$$

In the bank of phenomenological models analytical and numerical solutions of diffusion equations are stored, taking into account irreversible and reversible chemical reactions of the 1 st and 2 nd order between the gas and traps in the solid, various cases of parallel diffusion, dissociation, diffusion taking into account the exchange of diffusing gas between the diffusion channels, etc. In solutions to the diffusion equations various concentration profiles of the gas in the membrane, various concentration and time dependences of the diffusion parameters, etc. are considered.

The choice of an adequate phenomenological model of diffusion is made by means of standard methods of mathematical statistics, e.g. using the Fischer criterion (which consists in comparing the dispersity parameter $M_{2}$ of the curve with the remaining sum of deviations in the respective point of the curve).

A diffusion model is considered to be adequate when the difference between the experimental curve and the theoretically constructed curve corresponds to the normal distribution, and no drift of residues is observed.

It was shown in Fig.1, that the theoretical curve, computed under the assumption of the classical diffusion model (curve 4, Fig.1), does not fit well with the experimental curve (curve 3, Fig.1) and that there still are differences between the experimental and theoretical curves (curve 7, Fig.1). A systematic drift of residues from the base line was observed (curve 7, Fig.1), indicating that the classical diffusion mechanism is inadequate in this case.

From the linearized plot (curve 4, Fig.4) we have that the model of parallel diffusion is more suitable. The fitness of this diffusion model was proved by the coincidence of the theoretical and experimental curves (see curves 6 and 3, Fig.1, respectively). 
An increased number of experimental curves is stored in the bank of experimental data (BED) organized by us. The necessary condition for this storage is standardization of the experimental data. In the bank of experimental data both the shape of the experimental curve and the conditions of the measurements are stored. For a comparison of the curves the classical diffusion model is used as the reference model.

The condensed shape of any experimental curve can be determined using Eq. (11):

$$
F^{S}\left(U_{T}\right)=1-U_{T}\left\lfloor D_{\text {eff }} l^{-2}\left(t-t_{0}\right)^{-1}\right\rfloor
$$

where the $\mathrm{U}$ values are given.

The determination is based on an analysis of the drift of residues from the base-line and an evaluation of the relative deviation of $D$ from the average diffusion coefficient $D_{\text {eff }}$ calculated using experimental data of the whole curve. The service programmes for the computer treatment of experimental data are used.

The advantageous application of condensed information of the "diffusion map" should be mentioned here. The diffusion model is determined simply from the position of the point, which represents the whole experimental curve in the $\beta_{1}$ vs. $\beta_{2}$ plot. By means of the diffusion map, it is possible to forecast changes of the diffusion curve which may be caused by the influence of various effects on the system investigated. The relationship between various diffusion mechanisms can be established. Moreover, the existence of "white spots" on the diffusion map stimulates the search for mechanisms of gas diffusion in solids not yet discovered. A part of the diffusion map representing several diffusion mechanisms of gas permeability is demonstrated in Fig.3.

\section{Selection of a Physical Model of Gas Diffusion}

In some cases the analysis of the experimental curves did not give us a possibility for determining the diffusion mechanism. In these cases we investigated the dependences of the effective diffusion parameters (e.g. diffusion coefficient) on temperature, partial pressure of the diffusing agent, size of the sample, defectivity of the material, etc.

Mathematical formulae based on the respective physical model were used for the evaluation of the experimental results. Generally, with the classical diffusion mechanism the value of the diffusion coefficient should be independent of the method used for the diffusion measurement. In practice, however, such a dependence has often been observed.

For this reason, in the study of the diffusion behaviour of polymer membranes several independent methods are usually applied.

\section{Conclusion}

A large number of experimental techniques for diagnostics of polymer selective membranes have been suggested recently. Methods based on the diffusion of gases in the membranes represent a powerful tool for investigating dynamic properties of the membranes. The existing information obtained from experimental data as well as the phenomenological models of the diffusion mechanisms are stored in the computer information bank and can be used for the evaluation of experimental data. The computer programmes DIGS suggested give the possibility of a preliminary treatment of experimental results, and of an evaluation of the diffusion characteristics related to the adequate diffusion mechanism. 


\section{References}

1. A.A. Shviryaev and I.N. Beckman, in: Diffusion Phenomena in Polymers. Publ. House OICHF Academy of Sciences USSR (1985), 44-45.

2. I.N. Beckman and A.A. Sviryaev, Vestn. Mosk. Univ., Ser.2 Khim. 22 (1981), 467-471.

3. A.A. Shviryaev and I.N. Beckman, ibid., 22 (1981), 517-521.

4. I.N. Beckman, A.A. Shviryaev and V. Balek, Thermochim. Acta 104 (1986), 255-265. 